\title{
Effects of singing classes on pulmonary function and quality of life of COPD patients
}

\author{
Amanda Gimenes Bonilha' \\ Fernanda Onofre ${ }^{2}$ \\ Maria Lucia Vieira' \\ Maria Yuka Almeida Prado \\ José Antônio Baddini \\ Martinez' \\ 'Internal Medicine Department, \\ Medical School of Ribeirão Preto, \\ University of São Paulo, Ribeirão \\ Preto, São Paulo, Brazil; ${ }^{2}$ Music \\ Department, School of Arts and \\ Communications, University of São \\ Paulo, Ribeirão Preto, São Paulo, Brazil \\ Trial registered at ClinicalTrials.gov: \\ NCT 00500526
}

Correspondence: José Antônio Baddini Martinez

Internal Medicine Department, Avenida Bandeirantes 3900, CEP: | 4048-800,

Ribeirão Preto, São Paulo, Brazil

Tel +55 |6 3602253।

Fax +I 551636336695

Email jabmarti@fmrp.usp.br

\begin{abstract}
This study aimed to investigate the effects of weekly singings classes on pulmonary function parameters and quality of life (QoL) of COPD patients. Forty-three patients were randomized to weekly classes of singing practice, or handcraft work. They performed spirometry and completed maximal respiratory pressure measurements, evaluations of dyspnea, and the Saint George's Respiratory Questionnaire, before and after 24 training classes. A functional evaluation, immediately after 10 minutes of singing practice, was also performed at the end of the study. Fifteen subjects completed the study in each group. In comparison to controls the singing group exhibited transitory elevations on the dyspnea Borg scale $(\mathrm{p}=0.02)$, and inspiratory capacity $(p=0.01)$, and decreases of expiratory reserve volume $(p=0.03)$, just after a short session of singing. There was a significant difference on changes of maximal expiratory pressures in the comparison between groups at the end of training. While the control group showed deterioration of maximal expiratory pressure, the singing group exhibited a small improvement $(p=0.05)$. Both groups showed significant improvements of QoL in within group comparisons. We have concluded that singing classes are a well tolerated activity for selected subjects with COPD. Regular practice of singing may improve QoL, and preserve the maximal expiratory pressure of these patients.
\end{abstract}

Keywords: COPD; pulmonary function tests; breathing exercises

\section{Introduction}

Chronic obstructive pulmonary disease (COPD) is a disorder characterized by airflow limitation that is not fully reversible., ${ }^{1,2}$ Among several physiopathological effects, patients with COPD exhibit thoracic hyperinflation, decreases of maximal respiratory pressures, and dyspnea. ${ }^{1,2}$ As the disease progresses, limitations of daily living activities, poor performance status, and depression may add substantial harm to the overall quality of life (QoL) of these subjects., ${ }^{3,4}$

Singing, the act of producing musical sounds with the voice, is so basic to human beings that its origins are lost in antiquity, and predate the spoken language. It is well known that respiration has a key role in generating the voice, and it is an essential factor for singing as well., 5 The practice of singing involves strong and fast inspirations, followed by extended, regulated expirations. Singing, therefore, requires the presence of an accurate control of breathing. In addition, people who sing are practicing a particular type of respiratory exercise that repeatedly demands diaphragm contractions for full inspirations, followed by sustained contractions of expiratory muscles against semi-closed vocal cords during expirations. ${ }^{7,8}$ This training involving breathing control and respiratory muscle exertion has the potential of interfering with the pulmonary function of COPD patients. The regular practice of singing by patients with dyspnea might also lead to "desensitization" of breathlessness due to the development of better breathing coordination, and reduce the anxiety and fear associated with unpleasant respiratory sensations. Besides its possible effects on respiratory function, singing has 
been associated with improvements of mood, depression, and QoL in different settings, and may also induce the same kind of response in patients with chronic respiratory failure. . $^{9} 10,11,12,13,14$

Despite the therapeutic potential of singing for COPD, a MEDLINE search only revealed one published study on this topic, ie, a study by Engen (2005) which evaluated seven subjects after 12 singing classes given over 6 weeks. ${ }^{15}$ There were significant increases in the extent of counting and intensity of speech, and a change of breathing pattern from "clavicular" preponderance to a "diaphragmatic" one after the intervention. The results for QoL were inconclusive and the study, among other deficiencies, lacked a control group.

The objective of the present study was to investigate the effects of weekly singing classes on maximal respiratory pressures, spirometric measurements, and QoL of patients with COPD. It was our hypothesis that this practice could increase maximal respiratory pressures, decrease dyspnea, and improve QoL of COPD subjects on stable clinical conditions.

\section{Methods}

The subjects were invited to participate in the study during regular consultations at the University Hospital, or after answering a radio advertisement. Patients were required to have a diagnosis of COPD according to the Global Initiative for Chronic Obstructive Lung Disease criteria. ${ }^{1}$ All subjects were former smokers and had been in stable clinical conditions for at least two months before admission to the study. Patients with severe co-morbidities, still smoking, or using oxygen therapy were not included in the protocol. The investigation was approved by the Institutional Medical Ethics Committee, and the volunteers signed a written informed consent at the initial visit. All the evaluations and pulmonary function tests were performed at the Pulmonary Function Laboratory of the University Hospital of Ribeirão Preto, by the same group of researchers.

All patients initially completed the following set of tests; (i) spirometry with measurements of FVC, $\mathrm{FEV}_{1}, \mathrm{FEV}_{1} / \mathrm{FVC}$, IC, and ERV; (ii) measurements of maximal inspiratory and expiratory pressures at the mouth level ( $\left.\mathrm{PI}_{\max }, \mathrm{PE}_{\max }\right)$; (iii) arterial blood gases while breathing room air; (iv) dyspnea evaluation by the basal dyspnea index (BDI), and (v) assessment of QoL employing the specific instrument Saint George's Respiratory Questionnaire (SGRQ).

The volunteers were then randomized to a Singing Group or to a Control Group. The patients in the Singing Group were enrolled in weekly classes with an approximated duration of 1 hour, along at least 24 weeks. The classes were coordinated by a singing teacher and a physiotherapist. The patients participated in the classes as a group, and the activities included:

(i) Relaxation exercises of neck and upper limb muscles, conducted by a physiotherapist (about 5 minutes).

(ii) Singing related respiratory exercises conducted by a singing teacher (10 minutes). These exercises are part of regular singing teaching, and consisted of: performing fast, deep inspirations, followed by slow, full or interrupted expirations; performing fast and deep respiratory incursions, paying attention to the upper abdominal movements; generating breathing movements against, or with the help, of pressures generated by a hand placed on the upper abdominal region.

(iii) Vocalization exercises, lead by the singing teacher, as a preparation for singing (15 minutes). The patients loudly pronounced vowels as "le", "la", "mi", "mu". The patients also sang the melody of a familiar song using such vowels instead of actually singing the lyrics. Exercises of this kind are regularly employed by singers in order to warm-up before their artistic presentations.

(iv) Singing training of Brazilian folk songs, conducted by the singer teacher (30 minutes). In addition, the volunteers were also instructed to practice the folk songs at home for half an hour on at least two more days during the week.

The Control Group also attended a similar number of weekly classes. These lessons were coordinated by the same physiotherapist and by a different teacher of handcraft work. The activities of the Control Group included relaxation exercises as it has been previously described (about 5 minutes), and the execution of handcraft artwork such as paper folding, drawing, and collages (50 minutes). These patients were also routinely instructed to include some incomplete artwork or beginning a new one at home.

The final evaluation of the patients was performed after they had attended 24 classes. If episodes of acute exacerbations occurred, the final assessment was performed only after a minimum of one month of clinical stability, and the subject were required to have attended at least three sequential classes.

The components of the final evaluation were the same as those for the initial assessment, and were obtained on the same occasion, about 1 week after the last class. An additional set of tests was also performed on the same day. After a short resting period, the subjects performed 
vocalization exercises and singing in the upright position for 10 minutes. The new set of evaluations included spirometry, scoring of Borg dyspnea rating, and measurement of arterial oxygen saturation $\left(\mathrm{SaO}_{2}\right)$, obtained immediately before the beginning of the singing session, and 2 and 30 minutes after its interruption. In addition, Borg dyspnea rating and $\mathrm{SaO}_{2}$ were also recorded 5 minutes after the beginning of the singing exercise. Control group patients were submitted to the same type of tests, but instead of singing they remained silent in standing position for a comparable 10-minute period.

The spirometric data were all obtained in the sitting position using a Pulmonet Godard spirometer (Sensormedics, Bilthoven, The Netherlands), and the proceedings were performed according to the recommendations of the Brazilian Thoracic Association. ${ }^{16}$ The reference values of Crapo et al were employed for the calculation of spirometric results expressed as percentage of normality. ${ }^{17}$ Maximal respiratory pressures were measured using an MPG vacuum manometer (OEM Medical, Marshalltown, IA, USA). P $_{\max }$ values were measured starting on residual volume, while $\mathrm{PE}_{\max }$ was based on total lung capacity. The measurements of both pressures were made using at least 3 maneuvers with less than $10 \%$ variation, and the best value among them was chosen as the representative one. The equations of Neder et al were employed to express maximal respiratory pressures as percentage of predicted values. ${ }^{18}$ The arterial blood samples for gas analysis were always drawn from the radial artery with the individuals at rest and breathing room air. After collection, the material was promptly analyzed using a gas system analyzer (model 178; Corning, Medfield, MA, USA). A validated Portuguese version of the SGRQ was employed to measure QoL. ${ }^{19}$

As this was a pioneer investigation related to the effects of singing classes on pulmonary function of COPD patients, the numbers required to detect a difference within the primary outcomes were not predetermined. Based on a previous study that had found significant differences of maximal respiratory pressures following specific muscle training using 4 COPD groups containing 8 subjects, we opted for studying 2 groups of 15 patients each. ${ }^{20}$ All results are expressed as means and standard deviations. The results of the initial assessment are shown as absolute values and the results of the second evaluation are shown as the final values minus the initial ones $\left(\Delta_{\text {final-initial }}\right)$. The latter approach was also adopted to express the results of the acute test of singing exercise $\left(\Delta_{\text {during }}, \Delta_{2 \text { min }}\right.$, and $\left.\Delta_{30 \text { min }}\right)$. Groups were compared by the unpaired Student $t$ test. Comparisons of proportions between the two groups were made by chi-square tests. The comparisons between SGRQ scores obtained before and after intervention in the same group were performed by the paired t test. A p value $\leq 0.05$ was considered to be statistically significant.

\section{Results}

Seventy-eight patients were evaluated as potential candidates for the study. Thirty-five of them were excluded because they did not meet the inclusion criteria or because they refusal to participate in the protocol (Figure 1). Among the 43 patients who agreed to participate in the investigation, 23 were allocated to the Singing Group and 20 to the Control Group. A total of 13 patients discontinued the study, but at a proportion not significantly different between groups (Singing Group: 8; Control Group: 5). The reasons for abandoning the study in the Singing Group included non-medical causes (3) and non-pulmonary medical conditions (4). Only one patient of the Singing Group left the study due to repeated episodes of acute COPD exacerbation, and difficulties in regularly attending the classes. The reasons for dropping out of the Control Group were non-medical causes (4), and a non-pulmonary medical condition in one case. Fifteen patients concluded the entire protocol in both arms of the interventions. The final gender composition was similar in both groups, with a marked predominance of males.

The groups did not differ significantly regarding their basal clinical or functional features (Table 1). Although the mean QoL score of the Control Group was higher than that of the Singing Group, the difference did not reach statistical significance $(\mathrm{p}=0.06)$. In general, both groups could be classified as exhibiting moderate to severe obstructive disease, normal maximal respiratory pressures, and only mild gas exchange abnormalities.

The total number of missed classes did not differ significantly between groups (Singing Group: $50 \times$ Control Group: 57), nor did the number of episodes of non-attendance due to acute exacerbations (Singing Group: $4 \times$ Control Group: 6). The mean time for completing 24 classes was $28.2 \pm 1.8$ weeks for the Singing Group, and 28.8 \pm 2.4 weeks for the Control Group. Vocal exercises and singing popular songs were well tolerated by the patients. They did not complain of severe dyspnea, chest pain, regurgitation or dizziness, although a high prevalence of coughing and sputum expectoration was observed during the resting intervals.

The clinical and physiological responses of the subjects to the interventions are listed in Table 2. There was a 


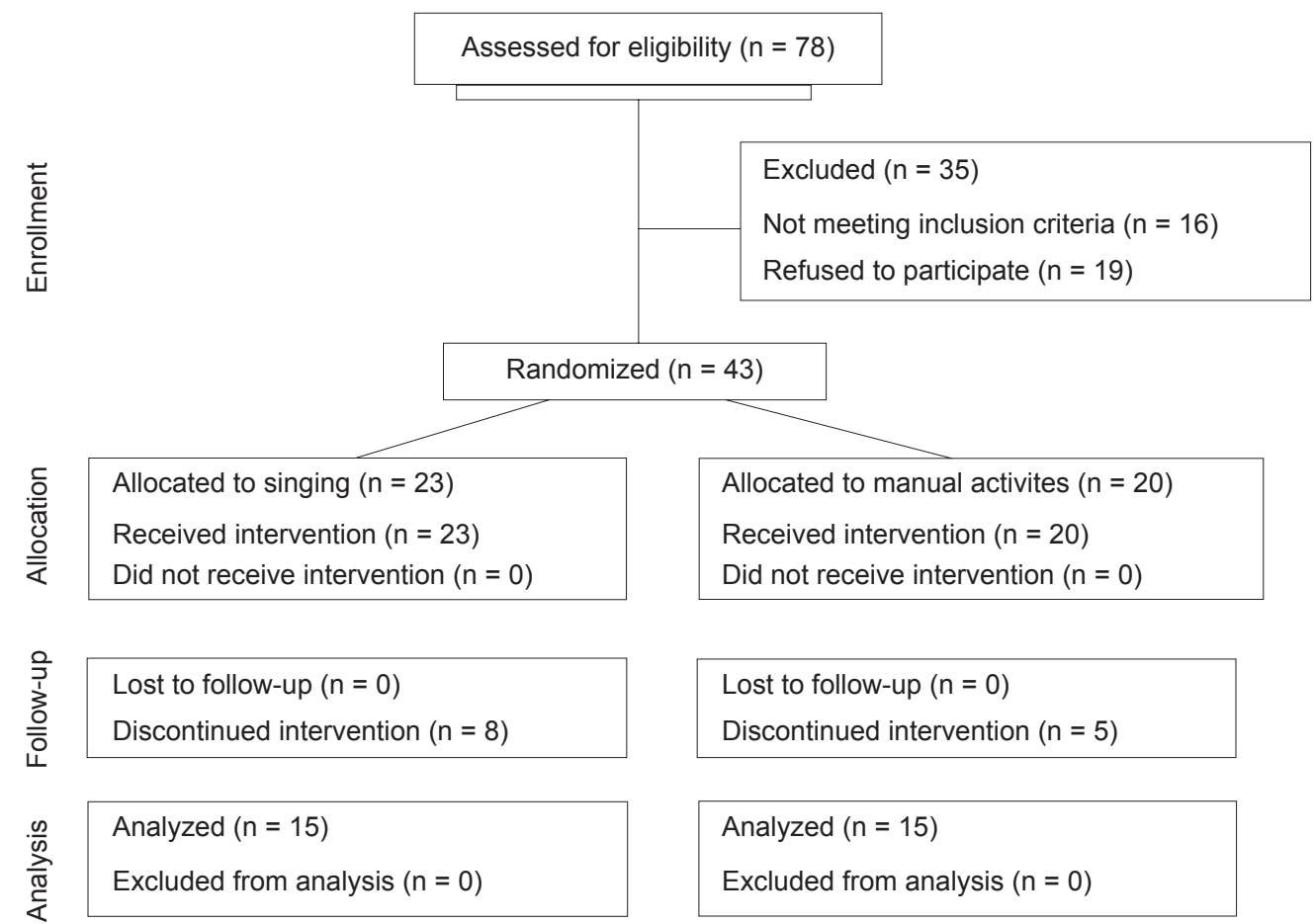

Figure I Consort diagram of the study.

statistically significant difference between groups only regarding the measurements of $\mathrm{PE}_{\max }$. While the Control Group exhibited a mean decrease in $\mathrm{PE}_{\max }$ close to $11 \mathrm{cmH}_{2} \mathrm{O}$, the Singing Group showed an increase of $3 \mathrm{cmH}_{2} \mathrm{O}$. It is also worth mentioning that, although the comparison of mean SGRQ variations between the groups was not significant, the $\Delta_{\text {(final-initial) }}$ reached at least 5 points in each group. In addition, intra-group paired $t$ tests comparing initial and final SGRQ scores revealed that both changes exhibited statistical significance (Singing Group: initial $=36.4 \pm 10.9 \times$ final $=30.5 \pm 9.6, p=0.001$; Control Group: initial $=45.7 \pm$ $14.3 \times$ final $=40.7 \pm 15.6, p=0.03$ ).

The acute responses to a short period of singing practice at the end of the period of singing classes are shown in Table 3. The singing practice led to a small but significant increase on the Borg dyspnea scale 2 minutes after the end of the exercise. A significantly higher $\mathrm{SaO}_{2}$ was also found during the act of singing. Finally, a bout of singing was associated with distinct effects on ERV and IC, detected 2 minutes after its interruption. While the Control Group showed an increase of ERV and a decrease of IC, the Singing Group had opposite outcomes.

\section{Discussion}

The present study showed that singing is a feasible practice among patients with moderate to severe COPD in stable clinical conditions. In addition, it has been shown here, for the first time, that singing may acutely promote small and transitory reductions of pulmonary dynamic hyperinflation, and to preserve $\mathrm{PE}_{\max }$ in the long run.

The patients tolerated well the respiratory efforts related to the singing classes and did not exhibit complaints during the lessons. A standard clinical-physiological evaluation for a short period of singing revealed that $\mathrm{SaO}_{2}$ and dyspnea scores increased during and just after the practice, respectively. The last finding is not unexpected and, most probably, is due to increases in the work of respiration. The changes of $\mathrm{SaO}_{2}$ could be secondary to hyperventilation during singing. However, the spirometric results performed immediately after singing suggest that vocal exercises may also induce transitory improvements in respiratory mechanics.

The patients of the Control Group showed a mean decrease in IC of $-0.08 \mathrm{~L}$ and a mean increase in ERV of $0.09 \mathrm{~L}$ after standing for 12 minutes. These values changed to $-0.10 \mathrm{~L}$, and $0.11 \mathrm{~L}$, respectively, after the subjects kept the same posture for 40 minutes. This suggests that the natural trend for silent COPD patients in the standing position is to progressively breathe at higher levels of FRC. The practice of singing for 10 minutes opposed this course. The mean IC of the Singing Group increased 0.14 L, and the mean ERV decreased $-0.01 \mathrm{~L} 2$ minutes after the patients stopped singing, respectively reaching $0.07 \mathrm{~L}$ and 
Table I Initial clinical features of the groups

\begin{tabular}{|c|c|c|}
\hline & Singing group & Control group \\
\hline $\mathrm{n}$ & 15 & 15 \\
\hline Male/Female & $12 / 3$ & $12 / 3$ \\
\hline Age (years) & $69.8 \pm 7.4$ & $73.6 \pm 7.5$ \\
\hline FVC (L) (\%) & $\begin{array}{l}2.42 \pm 0.57 \\
(84.3 \pm 28.3)\end{array}$ & $\begin{array}{l}2.70 \pm 0.61 \\
(96.1 \pm 24.1)\end{array}$ \\
\hline $\mathrm{FEV}_{1}$ (L) (\%) & $\begin{array}{l}\text { I.II } \pm 0.47 \\
(48.8 \pm 20.8)\end{array}$ & $\begin{array}{l}1.18 \pm 0.47 \\
(53.4 \pm 20.1)\end{array}$ \\
\hline $\mathrm{FEV}_{\mathrm{I}} / \mathrm{FVC}(\%)$ & $46.0 \pm 17.5$ & $43.0 \pm 10.7$ \\
\hline $\mathrm{ERV}(\mathrm{L})$ & $0.49 \pm 0.30$ & $0.70 \pm 0.33$ \\
\hline IC (L) & $2.11 \pm 0.66$ & $2.06 \pm 0.52$ \\
\hline $\mathrm{PI}_{\max }\left(\mathrm{cmH}_{2} \mathrm{O}\right)(\%)$ & $\begin{array}{l}87.7 \pm 22.2 \\
(92.2 \pm 20.4)\end{array}$ & $\begin{array}{l}75.3 \pm 26.4 \\
(82.1 \pm 25.9)\end{array}$ \\
\hline $\mathrm{PE}_{\max }\left(\mathrm{cmH}_{2} \mathrm{O}\right)(\%)$ & $\begin{array}{l}119.7 \pm 33.9 \\
(116.9 \pm 26.0)\end{array}$ & $\begin{array}{l}1 \mid 3.0 \pm 40.2 \\
(1 \mid 4.7 \pm 35.8)\end{array}$ \\
\hline $\mathrm{PaO}_{2}(\mathrm{kPa})$ & $9.4 \pm 1.2$ & $9.6 \pm 1.2$ \\
\hline $\mathrm{PaCO}_{2}(\mathrm{kPa})$ & $5.3 \pm 0.8$ & $5.1 \pm 0.4$ \\
\hline $\mathrm{P}(\mathrm{A}-\mathrm{a}) \mathrm{O}_{2}(\mathrm{kPa})$ & $3.9 \pm 0.8$ & $3.7 \pm 1.2$ \\
\hline BDI & $6.9 \pm 1.9$ & $6.1 \pm 1.8$ \\
\hline SGRQ & $36.4 \pm 10.9$ & $45.7 \pm 14.3$ \\
\hline
\end{tabular}

Abbreviations: BDI, basal dyspnea index; SGRQ, saint george's respiratory questionnaire.

$0.02 \mathrm{~L}, 28$ minutes later. The present results suggest that singing led the patients to breathe at lower FRC levels during and just after practice. This finding may reflect the occurrence of transient reductions in the extent of thoracic hyperinflation. Singing, in this context, could act promoting transitory changes in pressure-volume relationships of the respiratory system. It would work in a similar way as the controlled-breathing technique called active expiration. This technique involves the contraction of abdominal muscles during expiration, resulting in increased abdominal pressures. ${ }^{21}$ This lengthens the diaphragm of COPD patients close to its optimal size, and contributes to a better muscle function. In addition, active expirations would also increase the elastic recoil pressure of the diaphragm and rib cage. The release of this pressure after relaxation of the expiratory muscles could assist the next inspiration. ${ }^{22}$ Previous studies have shown that active expiration led to decreases of FRC and increases of trans-diaphragmatic pressures. ${ }^{23,24}$ The explanations for these findings include the reduction of thoracic volume and the improvement of the starting position of the diaphragm. It is likely that singing induces similar responses in COPD patients although, based on the present results, it appears to occur only in a temporary fashion. It is worth also to notice that a recent study suggests that laughter may promote reduction of static hyperinflation in severe COPD patients until 24 hours after the practice. ${ }^{25}$ Although the assessments of the acute effects of singing were performed only after a period of 24 classes, some patients who were also evaluated after 3 classes showed similar findings (data not shown). Therefore, these acute changes appear to be secondary to the act of singing itself, and not to the whole period of singing training. Besides, because of their transitory nature they, most probably, lack of clinical meaning.

The most important functional finding of this study was the positive influence of singing on $\mathrm{PE}_{\max }$. While the Control Group showed a decrease of $11.3 \mathrm{~cm} \mathrm{H}_{2} \mathrm{O}$, the Singing Group exhibited an increase of $3 \mathrm{~cm} \mathrm{H}_{2} \mathrm{O}$ in mean $\mathrm{PE}_{\max }$ at the end of the protocol. The first result is not totally

Table 2 Physiological responses under basal conditions after 24 singing classes

\begin{tabular}{|c|c|c|c|c|}
\hline & $\begin{array}{l}\text { Singing group } \\
\Delta_{\text {(final-initial) }}^{\text {b }}\end{array}$ & $\begin{array}{l}\text { Control group } \\
\Delta_{\text {(final-initial) }}\end{array}$ & p values ${ }^{a}$ & $\begin{array}{l}\text { Power of } \\
\text { the test (\%) }\end{array}$ \\
\hline FVC (L) & $-0.14 \pm 0.48$ & $-0.10 \pm 0.30$ & 0.76 & 24.1 \\
\hline $\mathrm{FEV}_{1}(\mathrm{~L})$ & $-0.03 \pm 0.31$ & $0 \pm 0.14$ & 0.76 & 35.6 \\
\hline $\mathrm{FEV}_{\mathrm{I}} / \mathrm{FVC}(\%)$ & $1.9 \pm 8.3$ & $1.5 \pm 2.9$ & 0.85 & 34.9 \\
\hline ERV (L) & $0.06 \pm 0.4$ & $-0.11 \pm 0.2$ & 0.13 & 79.5 \\
\hline IC (L) & $-0.09 \pm 0.3$ & $0.07 \pm 0.3$ & 0.14 & 66.3 \\
\hline $\mathrm{PI}_{\max }\left(\mathrm{cmH}_{2} \mathrm{O}\right)$ & $3.0 \pm 19.2$ & $-1.0 \pm 15.5$ & 0.54 & 30.1 \\
\hline $\mathrm{PE}_{\max }\left(\mathrm{cmH}_{2} \mathrm{O}\right)$ & $3.0 \pm 17.2$ & $-11.3 \pm 20.2$ & 0.05 & 90.1 \\
\hline $\mathrm{P}(\mathrm{A}-\mathrm{a}) \mathrm{O}_{2}(\mathrm{kPa})$ & $-0.4 \pm 0.8$ & $0.1 \pm 1.6$ & 0.22 & 19.2 \\
\hline BDI & $0.7 \pm 1.2$ & $0.3 \pm 1.7$ & 0.47 & 14.9 \\
\hline SGRQ & $-5.9 \pm 5.8$ & $-5.0 \pm 7.8$ & 0.72 & 5.4 \\
\hline
\end{tabular}

${ }^{a}$ Comparisons between groups by unpaired $\mathrm{t}$ test.

${ }^{\mathrm{b}} \Delta_{\text {(final-initial) }}:$ value after minus before classes.

Abbreviations: BDI, basal dyspnea index; SGRQ, saint george's respiratory questionnaire. 
Table 3 Acute physiological responses during and just after a singing exercise

\begin{tabular}{|c|c|c|c|c|c|}
\hline & & Singing group & Control group & p values $^{\mathrm{a}}$ & Power of the test (\%) \\
\hline \multirow[t]{3}{*}{ Dyspnea } & $\Delta_{\text {durin }}{ }^{\mathrm{b}}$ & $0.1 \pm 0.4$ & $-0.3 \pm 0.6$ & 0.14 & 93.1 \\
\hline & $\Delta_{2 \mathrm{mi}}{ }^{\mathrm{c}}$ & $0.5 \pm 0.7$ & $-0.3 \pm 0.6$ & 0.02 & 99.9 \\
\hline & $\Delta_{30 \min }{ }^{d}$ & $0.1 \pm 0.5$ & $-0.5 \pm 0.9$ & 0.07 & 95.2 \\
\hline \multirow[t]{3}{*}{$\mathrm{SaO}_{2}(\%)$} & $\Delta_{\text {during }}$ & $1.6 \pm 1.8$ & $0 \pm 1.2$ & 0.01 & 99.7 \\
\hline & $\Delta_{2 \min }$ & $0.4 \pm 2.0$ & $-0.4 \pm 1.3$ & 0.20 & 16.9 \\
\hline & $\Delta_{30 \min }$ & $-0.1 \pm 1.8$ & $-0.1 \pm 1.7$ & 0.92 & 4.1 \\
\hline \multirow[t]{2}{*}{ FVC (L) } & $\Delta_{2 \min }$ & $0.14 \pm 0.24$ & $0.04 \pm 0.20$ & 0.22 & 48.5 \\
\hline & $\Delta_{30 \min }$ & $0.13 \pm 0.35$ & $0.06 \pm 0.18$ & 0.52 & 4.5 \\
\hline \multirow[t]{2}{*}{$\mathrm{FEV}_{1}(\mathrm{~L})$} & $\Delta_{2 \min }$ & $0.04 \pm 0.16$ & $0.05 \pm 0.07$ & 0.83 & 0.07 \\
\hline & $\Delta_{30 \min }$ & $0.03 \pm 0.15$ & $0.04 \pm 0.07$ & 0.77 & 0.15 \\
\hline \multirow[t]{2}{*}{$\mathrm{FEV}_{1} / \mathrm{FVC}(\%)$} & $\Delta_{2 \min }$ & $-1.7 \pm 4.5$ & $0.64 \pm 3.9$ & 0.13 & 66.5 \\
\hline & $\Delta_{30 \min }$ & $-2.3 \pm 5.1$ & $0.24 \pm 2.7$ & 0.10 & 70.4 \\
\hline \multirow[t]{2}{*}{$\operatorname{ERV}(\mathrm{L})$} & $\Delta_{2 \min }$ & $-0.01 \pm 0.11$ & $0.09 \pm 0.14$ & 0.03 & 92.9 \\
\hline & $\Delta_{30 \min }$ & $0.02 \pm 0.34$ & $0.11 \pm 0.18$ & 0.25 & 12.1 \\
\hline \multirow[t]{2}{*}{ IC (L) } & $\Delta_{2 \min }$ & $0.14 \pm 0.25$ & $-0.08 \pm 0.18$ & 0.01 & 99.2 \\
\hline & $\Delta_{30 \min }$ & $0.07 \pm 0.34$ & $-0.10 \pm 0.19$ & 0.10 & 69.9 \\
\hline
\end{tabular}

${ }^{a}$ Comparisons between groups by unpaired $\mathrm{t}$ test.

${ }^{\mathrm{b}} \Delta_{\text {during }}$ : value during singing minus value just before singing.

${ }^{\mathrm{c}} \Delta_{2 \min }$ : value 2 minutes after singing minus value just before singing.

${ }^{\mathrm{d}} \Delta_{30 \text { min }}$ : value 30 minutes after singing minus value just before singing.

Abbreviation: $\mathrm{SaO}_{2}$, arterial oxygen saturation.

unexpected, since previous studies have shown a negative correlation between age and $\mathrm{PE}_{\max }$, both in healthy women and men. ${ }^{26,27}$ Even though the results of these articles do not completely support the finding of a so marked decrease on $\mathrm{PE}_{\max }$, the decline of $\mathrm{PE}_{\max }$ in patients of the Control Group should be a time-related physiologic descent, aggravated by superimposed deleterious effects of COPD itself. The act of singing demands higher respiratory work, mainly from the expiratory muscles, in order to generate extended musical sounds. Long, repeated contractions of the external and internal oblique, rectus, and transversus abdominis muscles occurring during singing, probably operated as a specific training for these elements. This type of training should be responsible for the observed prevention of losses of $\mathrm{PE}_{\max }$ in the Singing Group. The present results suggest that singing classes could be a practical and pleasant way of training expiratory muscles. Although the clinical value of training expiratory muscles is still debatable, recent data indicate that this approach may lead to increases of their endurance and strength, improvement of exercise performance, symptoms, and QoL in COPD patients..$^{28,29}$ Therefore, additional studies are needed to investigate the role of singing classes as a method for expiratory muscle training.
It is worth noting that the Singing Group also showed a mean $\mathrm{PI}_{\max }$ increase of $3.0 \mathrm{cmH}_{2} \mathrm{O}$, and the change of the Control Group was of $-1.0 \mathrm{cmH}_{2} \mathrm{O}$ only. A negative correlation between $\mathrm{PI}_{\max }$ and age has been previously described in women, but not in men. ${ }^{26,27}$ As most of the subjects enrolled in the present study were men, the minimal changes in $\mathrm{PI}_{\text {max }}$ of the Control Group after 28.8 weeks, is explainable.

The changes in the global SGRQ score did not significantly differ between the two groups. Nevertheless, both of them exhibited reductions of QoL mean score of at least 5.0 points. These decreases in scores reached statistical significance in a within-group analysis. An intervention that produces a change of 5.0 points in the SGRQ score may be classified as a slightly effective treatment. ${ }^{19,30}$ Therefore, both singing and handcraft artwork promoted comparable and meaningful improvements in overall QoL of COPD subjects evaluated by the SGRQ. The most marked changes in sections of the SGRQ were observed in Symptoms and Impact (data not shown). Several factors may contribute to poor QoL in COPD patients including respiratory symptoms, exercise limitations, depression, and lower sociability. The engagement in a regular pleasurable practice involving social interaction with health professionals and 
subjects with the same disease sharing similar problems and interests has the potential to positively influence the attitudes and perceptions of these patients. In this context, QoL may improve independently of the type of intervention implemented. As a result, it is possible that if the Control Group had not performed any activity, the positive effects of singing on QoL would have been demonstrated more clearly.

The investigators also observed that the patients coughed and eliminated a substantial amount of sputum just after ending the practice of vocal exercises or songs. Although this finding was not registered by employing a formal research protocol, it suggests that singing may also exhibits bronchial hygiene properties. The performance of prolonged and robust expirations has the potential to facilitate the mobilization of respiratory secretions towards the upper airways, eliciting the cough reflex. The improvement of $\mathrm{PE}_{\max }$ associated with singing could also contribute to better coughing. Additional studies aimed to adequately investigate these aspects are still necessary.

The present study exhibits fair number of limitations including small statistical power for some of the comparisons, meanly due to excessive variation in the results. As an example, while the analysis of the treatment effect on $\mathrm{PE}_{\text {max }}$ showed a test power of $90.2 \%$, the value for the comparisons of $\mathrm{PI}_{\max }$ and BDI were, respectively, of $30.1 \%$ and $14.9 \%$. Therefore, it can not be excluded the possibility that the enrollment of a greater number of subjects would evidence significant changes also in these parameters. Another criticism to be done is that the Singing Group has practiced not only singing during the classes, but additional respiratory and vocalization exercises as well. However, such respiratory and vocal exercises are important steps for a singer to improve his or her respiratory coordination. As a consequence, they are an essential part of the learning process of singing itself. Other deficiency of this research is that changes on respiratory pattern during the act of singing were not investigated, and the pulmonary volumes were not measured by body plethysmography. Besides, there was not the inclusion of patients with severe arterial hypoxemia in the present investigation.

Despite its limitations, the present study indicates that singing classes are an amusing, non-risky, and well-tolerated activity for selected subjects with COPD. Its regular practice may also improve QoL and preserve the $\mathrm{PE}_{\max }$ of these patients. Additional studies are recommended to better define the potential role of singing as a new tool for pulmonary rehabilitation.

\section{Disclosures}

The authors have no conflicts of interest to disclose.

\section{References}

1. Global Initiative for Chronic Obstructive Lung Disease. Global strategy for the diagnosis, management and prevention of chronic obstructive pulmonary disease. NHLBI/WHO workshop report. Available at:www. goldcopd.com. Accessed May 22, 2008.

2. Larsson K. Aspects on pathophysiological mechanisms in COPD J Intern Med. 2007;262(3):311-40.

3. Cully JA, Graham DP, Stanley MA, et al. Quality of life in patients with chronic pulmonary disease and comorbid anxiety or depression. Psychosomatics. 2006;47(4):312-9.

4. Carvalho NS, Ribeiro PR, Ribeiro M, Nunes MP, Cukier A, Stelmach R. Comparing asthma and chronic obstructive pulmonary disease in terms of symptoms of anxiety and depression. $J$ Bras Pneumol. 2007;33(1):1-6.

5. McCoy S. Breath management; gender-based differences in classical singes. Folia Phoniatr Logop. 2005;57(5-6):246-54.

6. Thomasson M, Sundberg J. Consistency of phonatory breathing patterns in professional operatic singers. $J$ Voice. 1999;13(4):529-41.

7. Hoit JD, Jenks CL, Watson PJ, Cleveland TF. Respiratory function during speaking and singing in professional country singers. $J$ Voice 1996;10(1):39-49.

8. Pettersen V. Muscular patterns and activation levels of auxiliary breathing muscles and thorax movement in classical singers. Folia Phoniatr Logop. 2005;57(5-6):256-77.

9. Grape C, Sandgren M, Hansson LO, Ericson M, Theorell T. Does singing promote well being ? An empirical study of professional and amateur singers during a singing lesson. Integr Physiol Behav Sci. 2003;38(1):65-74

10. Kenny DT, Faunce G. The impact of group singing on mood, coping and perceived pain in chronic pain patients attending a multidisciplinary pain clinic. J Music Ther. 2004;41(3):241-58.

11. Valentine E, Evans C. The effects of solo singing, choral singing and swimming on mood and physiological indices. Br J Med Psychol. 2001;74(1):115-20.

12. Kreutz G, Bongard S, Rohrmann S, Hodapp V, Grebe D. Effects of choir singing or listening on secretory immunoglobulin A, control, and emotional state. J Behav Med. 2004;27(6):623-35.

13. Clift SM, Hancox G. The perceived benefits of singing: findings from preliminary surveys of a university college choral society. JR Soc Health. 2001;121(4):248-56.

14. Takahashi T, Matsushita H. Long term effects of music therapy on elderly with moderate/severe dementia. J Music Ther. 2006;43(4):317-33.

15. Engen RL. The singer's breath: implications for treatment of persons with emphysema. J Music Ther. 2005;42(1):20-48.

16. Sociedade Brasileira de Pneumologia e Tisiologia. Diretrizes para testes de função pulmonar. J Pneumol. 2002;28(Supl 3):S1-S238.

17. Crapo RO, Morris AH, Gardner RM. Reference spirometric values using techniques and equipment that meet ATS recommendations. Am Rev Respir Dis. 1981;123(6):659-64.

18. Neder JA, Andreoni S, Lerario MC, Nery LE. Reference values for lung function tests. II. Maximal respiratory pressures and voluntary ventilation. Braz J Med Biol Res. 1999;32(6):719-27.

19. Camelier A, Rosa FW, Salim C, Nascimento OA, Cardoso F, Jardim JR. Using the Saint George's Respiratory Questionnaire to evaluate quality of life in patients with chronic obstructive pulmonary disease: validating a new version for use in Brazil. J Bras Pneumol. 2006;32(2):114-22.

20. Weiner P, Magadle R, Beckerman M, Weiner M, Berar-Yanay. Comparison of specific expiratory, inspiratory, and combined muscle training programs in COPD. Chest. 2003;124(4):1357-64.

21. Gosselink R. Controlled breathing and dyspnea in patients with chronic obstructive pulmonary disease (COPD). J Rehab Res Develop. 2003;40(Suppl 2):25-34. 
22. Erpicum B, Willeput R, Sergysels R, De Coster A. Does abdominal breathing below FRC give a mechanical support for inspiration? Clin Respir Physiol. 1984;20(5):117.

23. Reybrouck T, Wertelaers A, Bertrand P, Demedts M. Myofeedback training of the respiratory muscles in patients with chronic obstructive pulmonary disease. J Cardiopul Rehabil. 1987;7(3):18-22.

24. Casciari RJ, Fairshter RD, Harrison A, Morrison JT, Blackburn C, Wilson AF. Effects of breathing retraining in patients with chronic obstructive pulmonary disease. Chest. 1981;79(4):393-8.

25. Brutsche MH, Grossman P, Müller RE, et al. Impact of laughter on air trapping in severe chronic obstructive lung disease. Int J Chron Obstruct Pulmon Dis. 2008;3(1):185-92.
26. Black L, Hyatt R. Maximal respiratory pressures: normal values and relationship to age and sex. Am Rev Respir Dis. 1969;99(5):696-702.

27. Berry JK, Vitalo CA, Larson JL, Patel M, Kim MJ. Respiratory muscle strength in older adults. Nurs Res. 1996, 45(3):154-9.

28. Weiner P, Magadle R, Backerman M, Weiner M, Berar-Yanay N. Specific expiratory muscle training in COPD. Chest. 2003;124(2):468-73.

29. Mota S, Güell R, Barreiro E, et al. Clinical outcomes of expiratory muscle training in severe COPD patients. Respir Med. 2007;101(3): 516-24.

30. Ferrer M, Villasante C, Alonso J, et al. Interpretation of quality of life scores from the Saint George's Respiratory Questionnaire. Eur Respir J. 2002;19(3):405-13. 